\title{
Acoustic Models and Sonar Systems
}

\author{
Michael B. Porter
}

(Invited Paper)

\begin{abstract}
Acoustic models are used extensively in simulating sonar-system performance. Lately, such models have assumed a still more important role as they are incorporated in the signal processing algorithms. We review the basic model types and discuss their use in sonar systems.
\end{abstract}

\section{INTRODUCTION}

C OMPUTATIONAL ocean acoustics is usually concerned with solving the Helmholtz equation (or reduced waveequation) in an azimuthally symmetric environment:

$$
\nabla^{2} p+\frac{\omega^{2}}{c^{2}(r, z)} p=\frac{-\delta\left(r-r_{s}\right) \delta\left(z-z_{s}\right)}{r}
$$

where $c(r, z)$ is the ocean sound speed as a function of range and depth. In addition, $\omega$ is the angular frequency of the source which is located at the range/depth coordinate $\left(r_{s}, z_{s}\right)$. To complete the problem specification, we must also add boundary conditions at the ocean surface and bottom and a far-field radiation condition.

The goal is to solve for the response of the channel to the source, that is, to solve for the acoustic pressure $p(r, z)$. The transmission loss (in $\mathrm{dB}$ ) is then defined as $T L=-20 \log _{10}(4 \pi|p(r, z)|)$. Knowing the transmission loss between a source and reciever $(T L)$, how loud the source is $(S L)$, how loud the background noise is over which we seek to hear the source $(N L)$, and how directive the listening system is $(D I)$ we can then predict whether a source is detectable. This, of course, is expressed by the well-known sonar equation [1]:

$$
D T=S L-T L-N L+D I,
$$

where $D T$ is the detection threshold. When all the terms on the right add up to equal the detection threshold we begin to detect the source.

The various terms in the equation may be associated with computational acousticians $T L$, noise modelers $N L$, signal processors $D I$, and experimentalists $S L$. Practically speaking each component involves a few $\mathrm{dB}$ of uncertainty. On shore, in methodical intermodel comparisons, a fraction

Manuscript received April 8, 1993; revised May 4, 1993. This work was supported by the Office of Naval Research under Contract N00014-92-J, the Naval Research Laboratory under Contact N00014-91-J-2020, and the New Jersey Institute of Technology under Grant 421810.

The author is with the Department of Mathematics and Center for Applied Mathematics and Statistics, the New Jersey Institute of Technology, Newark NJ 07102.

IEEE Log Number 9212717

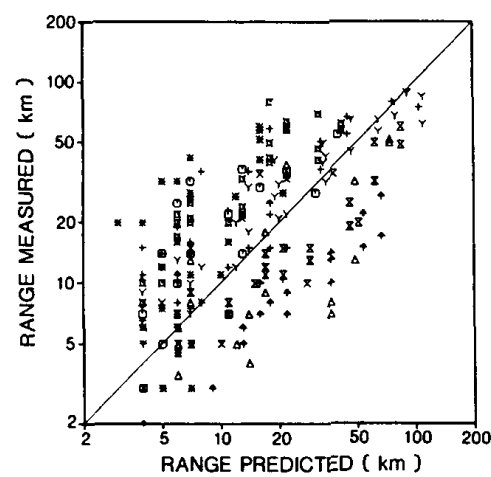

(a)

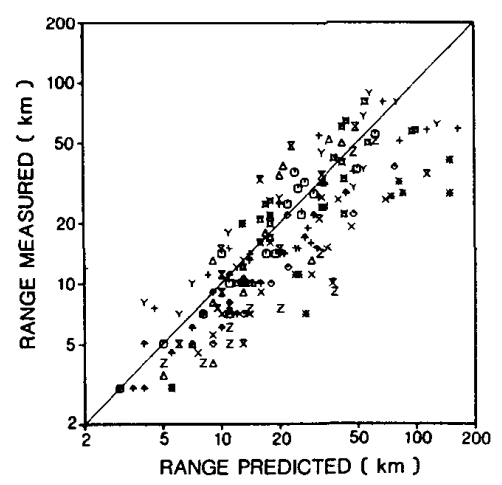

(b)

Fig. 1. (a) Comparison of an early propagation model to a more modern full-wave model (a) Colossus II-empirical model (1960), (b) SNAP-normal mode model (1980) (from [2])

of a $\mathrm{dB}$ error may be of interest in suggesting a more serious problem. At sea far greater errors may be induced by the common difficulty of just characterizing the bottom in coarse groups such as silt or sand.

Despite these difficulties there has been great progress in modeling transmission loss with a clear benefit in predicting sonar performance. This is illustrated in Fig. 1. This figure compares predicted versus measured detection ranges for two different generations of models. The models were applied to experimental data from 12 different sites in a blind fashion, that is, the environmental information was provided but not the answers ( $T L$ data)

The upper figure was obtained using the Colossus II model developed in the late 1950's. It uses empirical formulas for terms such as cylindrical spreading and volume attenuation. 
The lower figure was obtained using a modern normal mode model [2] and shows a much tighter clustering of points to the diagonal line (that represents perfect predictions). (These results are taken from a more complete report comparing many different modes [3].)

Depending on one's point of view this figure presents good or bad news. On the one hand, the efforts to improve the acoustic models have clearly had a real payoff. The results of the older, empirical model hardly correlate with the measured data. On the other hand, one can easily see points where the full-wave model has an error of a factor of two in predicted detection range.

Some would argue that the errors that remain in acoustic modeling are no longer due to shortcomings in the acoustic models but instead represent limits in our knowledge of the environment. On the other hand, our ability to survey the environment is also improving significantly. For instance, sidescan sonar systems are now able to provide detailed surveys of the ocean topography over wide areas in reasonable time. Progress in both oceanographic modeling and in remotely sensing the ocean structure is encouraging. In shallow water, the characteristics of the bottom are critical. However, on the time scales of interest to sonar systems, the ocean bottom is a static feature: In sites of particular interest its bathymetry and geoacoustic characteristics can be carefully measured.

In the remaining sections, we will review the basic types of acoustic models and demonstrate their application to conventional sonar simulation problems. We will also present examples of their use in more advanced signal processing applications.

\section{ACOUSTIC MODELS}

The acoustic models in widespread use today fall into four major classes: 1) ray theory, 2) spectral integration, 3) normal mode, and 4) parabolic equation. This classification is imperfect in that there are models that combine ideas from various approaches. Nevertheless, it is a useful starting point. In a short space, it is difficult to do justice to the various approaches. A more complete discussion is provided in [4].

The need for these various types of models stems from the diversity of applications. While one model may be capable of treating all the problems one encounters, usually at least some of the problems are more efficiently treated by another model. For instance, high-frequency problems are often most easily treated with ray models, range-dependent problems with parabolic equation models, and elastic wave problems with spectral integral models.

The starting point for all these models is the Helmholtz equation given in (1). This equation could be treated directly using finite-difference or finite-element methods. This is seldom done. Typically, such models require about 10 nodes per wavelength. Ocean acoustics problems are often thousands of wavelengths in range and tens of wavelengths in depth. The resulting linear systems of equations for the acoustic field at each node involve millions of unknowns. Thus, such techniques are not computationally very practical. The remaining approaches described in the next sections all make various simplifying assumptions.

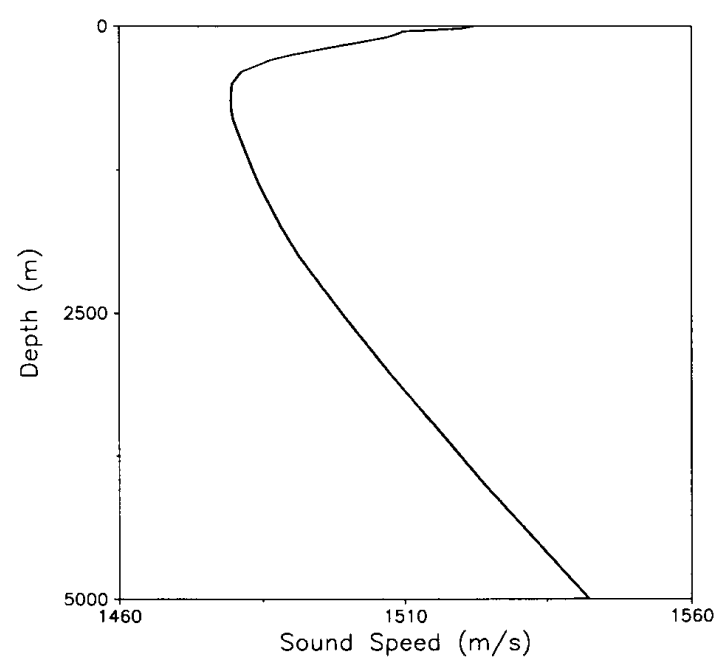

Fig. 2. Sound speed profile for the Pacific environment.

\section{A. Ray Theory}

Ray models were widely used in the early 1960 's. Today they are often viewed askance as more accurate fullwave models have become more popular. However, for highfrequency, broadband problems the ray methods may be hundreds of times faster. Rumors of their death are greatly exaggerated.

From a mathematical point of view, the ray equations are based on a high-frequency approximation. One seeks a solution of the Helmholtz equation of the following form:

$$
p(r, z)=e^{i k \phi(r, z)} \sum_{j=0}^{\infty} A_{j}(r, z) \frac{1}{(i k)^{j}}
$$

where $k=\omega / c_{0}$ and $c_{0}$ is a reference sound speed. This gives an infinite sequence of equations for the functions $\phi(r, z)$ and $A_{j}(r, z)$ :

$$
\begin{aligned}
(\nabla \phi)^{2} & =-c_{0}^{2} / c^{2}(r, z), \\
2 \nabla \phi \cdot \nabla A_{0}+(\Delta \phi) A_{0} & =0, \\
2 \nabla \phi \cdot \nabla A_{j}+(\Delta \phi) A_{j} & =-\Delta A_{j-1}, \quad j=1,2, \cdots
\end{aligned}
$$

The equation for $\phi(r, z)$ is known as the eikonal equation (after the Greek eikonos for image or icon). The equations for $A_{j}$ are known as the transport equations. The eikonal equation is solved by introducing a family of curves (rays) which are defined by being perpendicular to the level curves (wave-fronts) of $\phi(r, z)$. One finds that the rays satisfy:

$$
\begin{array}{ll}
\frac{d r}{d s}=c \rho(s), & \frac{d \rho}{d s}=-\frac{1}{c^{2}} \frac{d c}{d r}, \\
\frac{d z}{d s}=c \zeta(s), & \frac{d \zeta}{d s}=-\frac{1}{c^{2}} \frac{d c}{d z},
\end{array}
$$

where $(r(s), z(s))$ is the trajectory of the ray in the rangedepth plane and $(\rho(s), \zeta(s))$ is the local tangent vector to the ray. Of course, to solve these equations we need initial conditions. These are that the rays originate from the source with a user-specified takeoff angle.

As an example we consider a deep-water Pacific environment shown in Fig. 2. We place the source at a depth of 25 




Fig. 3. Ray trace for the Pacific environment.

$\mathrm{m}$ and then solve the ray equations with a sequence of ray takeoff angles varying over $\pm 25^{\circ}$. The resulting ray fan is shown in Fig. 3. Dotted lines have been used to show the bottom bounce paths.

This plot is only the skeleton of the acoustic field; to obtain the associated pressure field we must proceed further. However, this ray trace is often the most important product of a ray model. As mentioned above, other techniques can give more accurate transmission loss figures; however, they do not readily provide this simple graphical ray picture showing the important energy paths.

If we do wish the complete pressure field we must use these rays to solve the eikonal for the phase along the ray. The phase function $\phi(r, z)$ is given by a simple integral:

$$
\phi(s)=\int_{0}^{s} \frac{c_{0}}{c\left(s^{\prime}\right)} d s^{\prime}
$$

The ray coordinates are also useful for solving the transport equations for $A_{i}(r, z)$. Not liking to solve an infinite number of such equations one normally just solves for the leading term $A_{0}(r, z)$. The remaining terms have increasing powers $(i k)^{j}$ in the denominator and one argues that for sufficiently high frequency, they are negligible. Formally, the leading term $A_{0}$ satisfies a simple differential equation along the ray. In practice, the amplitude is often obtained from the rays themselves by calculating the spread between two adjacent rays.

Ray models have well-known shortcomings stemming from the high-frequency approximation inherent in their derivation. Basically, wherever there is a transition in the field, it occurs abruptly in the ray result while a correct full-wave solution would show a gradual change on the scale of a wavelength.

A feature of ray models is that they can be adapted to broadband problems with minor additional computational cost. The source waveform is simply propagated down the ray with a delay equaling the travel time and attenuated based on the spreading of the ray tube. (A simple $\mathrm{dB} /$ wavelength attenuation is normally added to account for volume attenuation.) All the rays arriving at a single point (eigenrays) yield a different arrival since usually each ray has a different travel time. This simple picture is only clouded by the need to introduce $90^{\circ}$ phase shifts when the ray crosses through caustics.

Ray models have been developed to a high degree but unfortunately the journal literature gives little insight into the techniques used in state-of-the-art programs. A fairly complete summary is provided in [4].

\section{B. Spectral Integral Methods (Fast-Field Program)}

Spectral integral and normal mode models are closely related. Both models assume a stratified ocean (no range dependence) and take advantage of this for speed. We must hastily mention that they can be extended to range-dependent cases in various ways.

With the assumption that there is no range variation it is useful to view the field in range as a sum of sines and cosines, i.e. a Fourier series. As often happens for problems on infinite domains the series involves a continuum of sines and cosines with an arbitrary horizontal wavenumber-the discrete sum becomes an integral. Finally, because the problem is in cylindrical coordinates the proper range functions are actually Bessel functions. These may be regarded as sines and cosines with cylindrical spreading.

Mathematically we start by applying a Fourier-Bessel transform to the Helmholtz equation given in (1):

$$
G(k, z)=\int_{0}^{\infty} p(r, z) J_{0}(k r) r d r
$$

which leads to

$$
\begin{aligned}
\frac{d^{2} G}{d z^{2}}+\left(\frac{\omega^{2}}{c^{2}(z)}-k^{2}\right) G & =\delta\left(z-z_{s}\right) \\
G(0) & =0, \quad \frac{d G}{d z}(D)=0 .
\end{aligned}
$$

We have included simple boundary conditions to illustrate the form. In practice, the lower boundary at $z=D$ is usually modeled as a half-space leading to a slightly more complicated equation. Solving the above depth-separated equation is the key numerical problem in a spectral integral code.

The solution of this boundary value problem yields $G(k, z)$ and the final pressure is then computed using the inverse Fourier-Bessel transform as

$$
p(r, z)=\int_{0}^{\infty} G(k, z) J_{0}(k r) k d k .
$$

This is the so-called spectral integral representation of the solution. As discussed above, we may view this integral as a sum of sines and cosines with a cylindrical spreading term. To make this formal, we use a large-argument approximation to the Bessel function to obtain:

$$
p(r, z) \approx \frac{e^{i \pi / 4}}{\sqrt{2 \pi r}} \int_{0}^{K} G(k, z) \sqrt{k e}^{i k r} d k .
$$

Here we have also ended the integral at some upper limit $K$ usually chosen a little above $\max (\omega / c(z))$. Beyond this point the integrand decays exponentially and does not make 


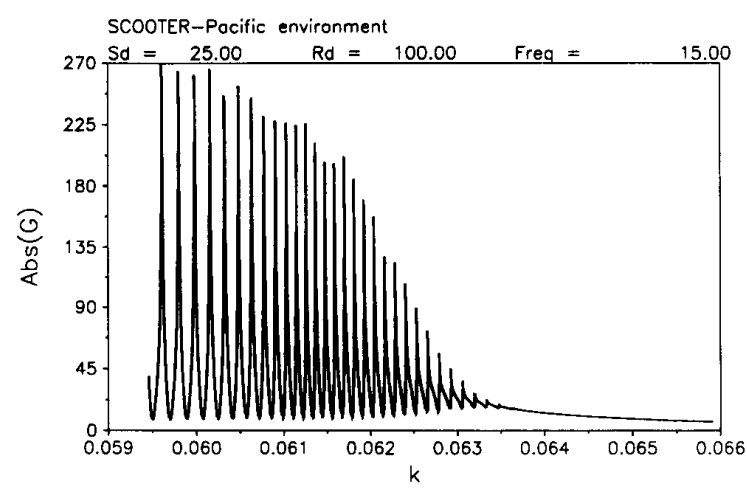

Fig. 4. Kernel for the Pacific environment.

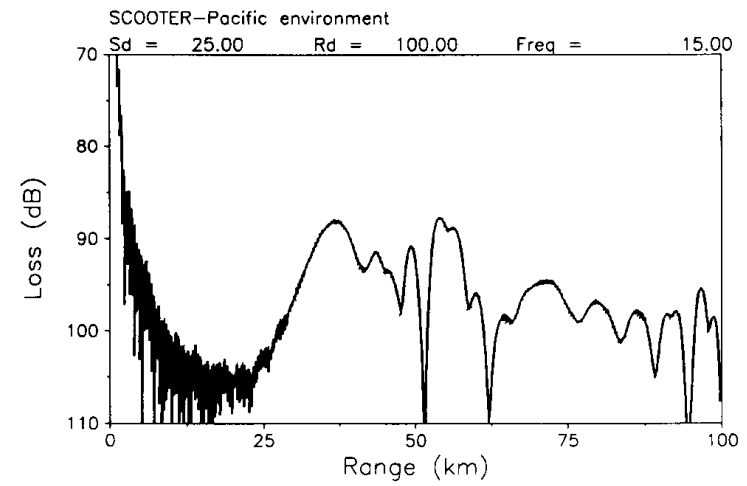

Fig. 5. Transmission loss for the Pacific environment.

a significant contribution. This may be seen in Fig. 4, where we have plotted the integrand for a $15 \mathrm{~Hz}$ source at depth 25 $\mathrm{m}$ and a receiver at depth $100 \mathrm{~m}$. The integrand represents the spectrum of the field. Performing the integral in (10) we recover the actual field versus range as illustrated in Fig. 5. This integral actually assumes the form of a Fourier transform. Thus, when there are many ranges it is efficient to evaluate it using an FFT. This technique was introduced by DiNapoli [5]. The model that combined an FFT with the spectral integral formulation was called the Fast-Field Program (FFP).

Since the FFP approach is based on a standard Fourier transform many of the numerical issues follow directly from standard filtering theory. Time corresponds to range and frequency to wavenumber. Long range calculations therefore require fine wavenumber sampling. Fine sampling in range is obtained by increasing the upper wavenumber limit, $K$ (or by zero-filling). When the spectrum is chopped abruptly as we did in Fig. 4 high-frequency ringing (Gibbs phenomenon) occurs that can be seen in Fig. 5. This ringing can be eliminated by tapering the kernel using, for instance, a Hann or Hamming window [6].

For range-independent problems the spectral integral result is typically taken as an exact solution. The key approximation is that of using the far-field approximation to the Hankel function. This approximation is accurate to a fraction of a $\mathrm{dB}$ beyond a couple of wavelengths from the source.
There are several acoustic codes based on this spectral integral representation. Probably the most widely used model is SAFARI [7]. It allows complicated multilayered elastic sediments, broadband and directive sources, and, interfacial roughness.

\section{Normal Modes}

If we look back at the spectrum shown in Fig. 4, we see that it has a spiky character. The spectrum is even spikier than this plot would suggest since it is plotted a slight distance off the real-axis to avoid the nearly singular points. Thus, the spectrum is really dominated by a finite number of discrete spatial 'resonances'. These correspond to the normal modes of the channel.

In the method of normal modes we solve for the frequencies of these spatial resonances. From a mathematical point of view there are two ways of deriving the modal solution. We may use methods from complex variable theory and replace the spectral integral by a sum of residues. Alternatively, if the boundary conditions are simple we can derive the modal expansion using separation of variables. We shall follow the latter approach.

Again one begins with the Helmholtz equation and seeks a solution as a sum of normal modes:

$$
p(r, z)=\sum_{j=1}^{\infty} Z_{j}(z) R_{j}(r) .
$$

Substituting the above form into the Helmholtz equation one obtains:

$$
\begin{aligned}
\frac{d^{2} Z_{j}}{d z^{2}}+\left(\frac{\omega^{2}}{c^{2}(z)}-k_{j}^{2}\right) Z_{j} & =0, \\
Z_{j}(0) & =0, \quad \frac{d Z_{j}}{d z}(D)=0,
\end{aligned}
$$

which is identical to the spectral integral form of (8), apart from lacking the forcing term on the right-hand side. The above equation has an infinite number of solutions that are like modes of a vibrating string. The modes are characterized by a mode shape function $Z_{j}(z)$ and a horizontal propagation constant $k_{j}$ (analogous to a frequency of vibration).

The range functions, $R_{j}(r)$ are found to satisfy,

$$
\begin{aligned}
& \frac{1}{r} \frac{\delta}{\delta r}\left(r \frac{\delta R_{j}}{\delta r}\right)+k_{j}^{2} R_{j}=-Z_{j}\left(z_{s}\right) \frac{\delta(r)}{r} \\
& \frac{1}{r} \frac{d}{d r}\left(r \frac{d R_{j}}{d r}\right)+k_{j}^{2} R_{j}=-Z_{j}\left(z_{s}\right) \frac{\delta(r)}{r}
\end{aligned}
$$

which has the solution $R_{j}(r)=\frac{i}{4} Z_{j}\left(z_{s}\right) H_{0}^{(1)}\left(k_{j} r\right)$. Putting all of this together, one finds that,

$$
p(r, z)=\frac{i}{4} \sum_{j=1}^{\infty} Z_{j}\left(z_{s}\right) Z_{j}(z) H_{0}^{(1)}\left(k_{j} r\right)
$$

or, using the asymptotic approximation to the Hankel function,

$$
p(r, z) \approx \frac{i}{\sqrt{8 \pi r}} e^{-i \pi / 4} \sum_{j=1}^{\infty} Z_{j}\left(z_{s}\right) Z_{j}(z) \frac{e^{i k_{j} r}}{\sqrt{k_{j}}} .
$$






Fig. 6 shows selected modes for the Pacific environment. The source frequency for this case was chosen to be $100 \mathrm{~Hz}$. The first group of modes corresponds to a family of ray paths that are completely trapped (refracted) within the SOFAR duct. Modes 1 and 10 are examples of such modes. The higher order modes penetrate further towards the ocean boundaries.

The second group of modes corresponds to rays that strike the ocean surface but refract away from the ocean bottom. Mode 80 is an example of this group. Typically, these modes would be lossier since they lose some energy when reflecting off the rough ocean surface.

The last group of modes involves ray paths that are so steep that the rays reflect off both the surface and bottom These modes tend to be the lossiest since they experience both surface and bottom bounce losses. Mode 160 is an example of this class.

The various loss mechanisms are accounted for by making the sound speed complex. As a result the horizontal wavenumbers $k_{j}$ have a positive imaginary part and therefore decay exponentially with range. As discussed above, the higher order modes are typically the lossiest. As such one computes only a finite number of the lower order modes.

Once the modes are calculated we can rapidly add them up to construct an image of the acoustic field. In Fig. 7 we have done this using a source depth of $25 \mathrm{~m}$. Note how the modes sum up to form the convergence zone pattern that we previously saw in the ray trace. In the near field we see a common Lloyd mirror pattern appearing as a series of beams emanating from the source. This pattern is due to constructive and destructive interference between the source and its reflection in the ocean surface. The beam that is launched at a shallow angle refracts to form the convergence zone pattern. The steeper beams reflect off the ocean bottom using significant energy in the process.

In the far-field the boundary losses tend to strip off the higher order modes. However, in the near field we must retain many modes to accurately account for the steep angle paths. An extra complication is that mode codes often use a trick

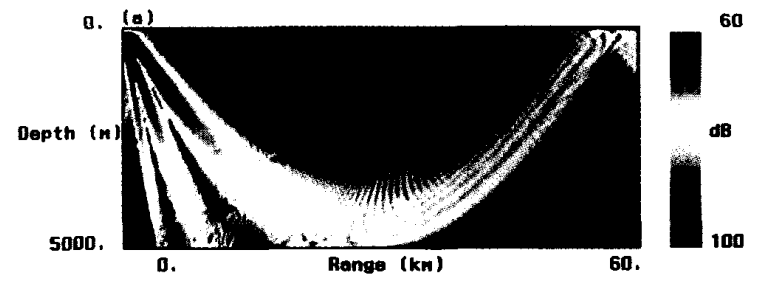

Fig. 7. Transmission loss for the Pacific environment.

in which all sediment and ocean volume attenuation is first removed. With this done, the modes divide into two distinct classes (trapped and leaky modes) depending on whether their corresponding rays are steep enough to travel into a lower halfspace (basement). The advantage of this approach is that the trapped modes are purely real (no loss) which simplifies the finding of eigenvalues. A loss factor is then added after the fact based on the attenuation along the ray paths associated with the modes. The leaky modes have complex wavenumbers and therefore require special root finders; they are simply ignored.

The means that such mode codes have a hardwired limit on how many modes they can include. This limits their accuracy to longer ranges. Obviously, the range at which such models are valid depends on the required accuracy and details of the environment but a rule of thumb is that they are appropriate at ranges greater than 10 water depths.

Note the complementary nature of normal mode and spectral integral modes. At longer ranges the modal calculation gets easier: Fewer modes need to be included. With spectral integral codes we must calculate the integrand at more points in its spectrum to obtain the field at long ranges. As such, an FFP model is often preferable for near-field calculations and a normal mode model for long ranges.

Like the other model types, there are many existing codes to use from. The most widely used are KRAKEN [8] and SNAP [2] which are based on an identical finite-difference algorithm [9]. 


\section{Parabolic Equation Modeling}

The parabolic equation was introduced in underwater acoustics in 1973 by Tappert and Hardin. In the PE derivation one factors the Helmholtz equation into right and left traveling wave equations that involve operator square roots and are formally pseudo-differential operators. However, as an introduction to the method we follow the simpler route of the original derivation, seeking a solution of the Helmholtz. equation in the form,

$$
p(r, z)=u(r, z) H_{0}^{(1)}\left(k_{0} r\right)
$$

where $k_{0}$ is a reference wavenumber. Substituting into (1) one finds,

$$
u_{r r}+2 i k_{0} u_{r}+k_{0}^{2}\left(n^{2}-1\right) u+u_{z z}=0 .
$$

At this point, one discards the first term to obtain the parabolic equation:

$$
u_{r}=i k_{0} \frac{n^{2}-1}{2} u+\frac{i}{2 k_{0}} u_{z z} .
$$

This latter step is justified assuming weak range-dependence and narrow angle propagation, i.e., when the dominant energy comes from rays propagating nearly horizontally.

The advantage of the parabolic equation over the original Helmholtz equation is that the PE can be solved by a straightforward march in range which requires much less computational effort. From a numerical point of view, this range marching is typically implemented using either standard finitedifference techniques [10] or using a fast Fourier transform as in the so-called "split-step" method [11].

In problems with strong range-dependence the PE method is generally the method of choice. The PE method, however, has several approximations. These approximations introduce errors that increase as the corresponding ray angle increases. However, a great deal of work has been done in the last 10 years to construct higher-angle PE's so that now the angle limitation is seldom a problem. This increased accuracy generally comes at the expense of computer time. For more details on modern PE's we refer the reader to Collins [12] and references therein.

\section{E. Three-Dimensional Acoustic Modeling}

A simple way to obtain a 3-D acoustic prediction is to run a 2-D model for a sequence of bearings. This approach is sometimes called $N \times 2-D$ or 2.5-D modeling. It neglects the bending of ray paths in the horizontal plane; however, these effects are often negligible. Of course, full 3-D modeling is also possible; ray and PE approaches have a direct generalization that poses no major theoretical problems. However, even with the geometric growth in computing power, full 3-D calculations are still impractical for the operational environment without some simplifying assumptions.

A widely used approach to 3-D modeling is based on normal modes [13]. To begin the calculation, the environment is divided into a triangular grid as illustrated in Fig. 8. This particular problem involves propagation across a 600 $\mathrm{km}$ square section of the Gulf Stream. The grid has been



Fig. 8. Triangulation of the Gulf Stream environment.

constructed to echo the principal features of the problem including the meadering Gulf Stream, cold core eddies, and a seamount. The grid lines have been colored to show cold (blue) and warm (red) water.

This scheme minimizes the number of triangles needed; however, for production runs it is generally more convenient to construct a regular triangular grid conforming to standard environment data bases such as BLUG (for bottom loss characteristics) and DBDB (a digital bathymetric data base).

Once the grid is constructed, the normal modes are calculated for each node using the sound speech, bottom depth and bottom impedance corresponding to the latitude/longitude of the nodal point. This is normally the most time-consuming part of the calculation; however, on modern workstations hundreds of such mode sets can be calculated in less than an hour for low- to midfrquencies.

Finally, a source position is selected and the field is calculated on a fan of radials emanating from the source. Along each radial the adiabatic mode formula gives the following approximation for the field:

$$
\begin{array}{r}
p(r, z, \theta)=\frac{i}{4} \sqrt{\frac{2}{\pi}} e^{i \pi / 4} \sum_{m=1}^{M(r, \theta)} Z_{m}\left(z_{s} ; 0,0\right) \\
\cdot Z_{m}(z ; r ; \theta) \frac{e^{i \int_{0}^{r} k_{m}(s, \theta) d s}}{\sqrt{k_{m}(r, \theta) r}} .
\end{array}
$$

The local modes $Z_{m}(z ; r, \theta)$ at a given range $r$ along the bearing angle $\theta$ are computed using linear interpolation within each triangular element.

The resulting transmission loss is shown in Fig. 9. This plot shows three orthogonal surfaces corresponding to TL for 1) a fixed receiver depth, 2) a fixed bearing, and 3) a fixed range. The slice for a fixed bearing clearly shows a typical deep water convergence zone pattern. The disc taken at a receiver depth of $400 \mathrm{~m}$ shows the acoustic shadow generated by the Gulf Stream. A smaller shadow is also generated by one of the eddies.

An important characteristic of this model is that if the source is moved a new 3-D $T L$ plot can be calculated in 




Fig. 9. Transmission loss for the Gulf Stream environment.

a few minutes. Most of the computational work is done in calculating the mode set at each nodal point; very little work is required to perform the modal sums for computing the acoustic field. This makes the model especially attractive for problems where the arrays and the sources are moving, for example for studying optimal deployment of ships in a task force. Indeed, the acoustical calculations are so rapid that the real computational problem is one of optics: the rendering and visualizing the massive $3-\mathrm{D}$ acoustic data sets.

Such 3-D acoustic models require a great deal of environmental information. In the long term one imagines that oceanographic data may be provided by combining ocean circulation models, mixed layer models and satellite measurements. Detailed bathymetric data will be available from side-scan sonar systems. Ocean bottom reflectivity data will be available from high-quality bottom loss data bases obtained by combining improved bottom inversion methods with the human insight of seafloor geologists and geophysicists.

The reader scrutinizing the detailed fringes in the 3-D $T L$ plots is certainly deluding himself about their reliability. However, even without such detailed 3-D environmental data the 3-D acoustic models can provide very useful information. We can clearly see the shadows of major oceanographic features and plan array deployment accordingly without placing tremendous confidence in the accuracy of the SSP data. Similarly, an optimal array location can often be determined knowing only that the bottom in one area is more or less reflective than that of another area. The actual $\mathrm{dB}$ loss per reflection may not be critical.

\section{APPLiCATIONS IN SONAR Systems}

Acoustic models are used in a variety of ways in connection with sonar systems. At their most mundane (but important) they are used like a weather forecaster to predict the sonar range of the day. In such cases, the detailed structure of the peaks and nulls in the transmission loss plots is of little relevance. The range of the day may in turn be used to suggest deployment patterns for air-deployed sonobuoys or, on a larger scale, for surface ships in a naval task force. Besides the lat/long positioning of hydrophone arrays, depth positioning is also a critical factor. This is discussed in more detail in [14]. [15].

In the last 10 years research has accelerated on sonar systems where the acoustic model is an integral part of the sonar system. The key point in such systems is that the refractive behavior of the ocean distorts the sound and as a result the source is out of focus. An acoustic model then provides the electronic lens to correct for this array myopia (or more precisely ametropia). Besides the refractive effects there are also the multiple reflections off the nearly perfect mirror of the ocean surface and the murkier but still reflective mirror at the ocean bottom. Again, the acoustic model can be used to find the true source in this house of acoustic mirrors. Some approaches are described below.

\section{A. Matched-Field Processing}

Let us first review traditional source localization schemes based on plane-wave beamforming. The array elements are phased to generate a beam in a particular look-direction. The 
beam is then swept across the ocean to locate the source. Let us review the equations for this process. We suppose the array is being steered to a particular look direction $\theta$. The appropriate steering vector has elements

$$
\boldsymbol{e}_{i}(\theta)=e^{i k z_{i} \sin \theta}
$$

which corresponds to the field that would be received on the array due to a plane wave incident at an angle $\theta$. Here, $k=\omega / c$ is the wavenumber. The coordinate $z$ measures distance along the array.

The field actually received by the array is a data vector denoted by $\boldsymbol{d}$. In conventional beamforming the steering angle $\theta$ is varied over a sweep of angles. At each angle we then compare the steering vector to the actual field measured by the array. When the steering vector most closely matches the data vector we claim to have identified the source bearing.

Of course, there are many ways to compare the steering and data vectors. The standard approach is to use a dot product:

$$
P(\theta)=\left|d^{*} e(\theta)\right|^{2}=e^{*} d d^{*} e .
$$

Often this is written

$$
P(\theta)=e^{*} R e
$$

where $R=\boldsymbol{d}^{*}$. We will call this the unnormalized power. Typically we have many observations $d_{l}$ of the field received on the array. We may then elect to average the data to form an improved estimate of $R$ given by $(1 / L) \sum_{l=1}^{L} \boldsymbol{d}_{l} \boldsymbol{d}_{l}^{*}$ where $L$ is the total number of frames. The matrix $R$ is the covariance matrix or cross-spectral matrix.

This scheme is sensible for horizontal arrays seeking targets in a horizontal plane; however, for a vertical array the effects of beam reflection by the surface and bottom are important. Furthermore, the ocean medium is refracting and therefore distorts a plane-wave field into a curved wavefront. As such, we must reassess the value of steering the array using planewave vectors.

To see how strong these effects are, we simulate a vertical array of 21 hydrophones positioned in the water column from $1000 \mathrm{~m}$ to $2000 \mathrm{~m}$ in depth. We steer the beam in the broadside direction $(\theta=0)$ and observe the beam response $P(r, z \mid \theta=0)$ as the source is moved over range and depth. To simulate the beam response we need an acoustic model. As a point of reference, we first remove the top and bottom ocean boundaries and use an isovelocity ocean. This gives a free space problem for which the acoustic model is especially simple. The field on the $i$ th phone is simply given by a spherical wave:

$$
d_{i}(r, z)=\frac{e^{i k \cdot R_{i}}}{R_{i}}
$$

where $R_{i}$ is the slant range from the source at $(r, z)$ to the hydrophone at $\left(r_{i}, z_{i}\right)$ :

$$
R_{i}=\sqrt{\left(r-r_{i}\right)^{2}+\left(z-z_{i}\right)^{2}} .
$$

In the following simulations we will use a $15-\mathrm{Hz}$ source. The unnormalized beam response for the free space case is shown in Fig. 10. We see the main lobe steered to the horizontal and a
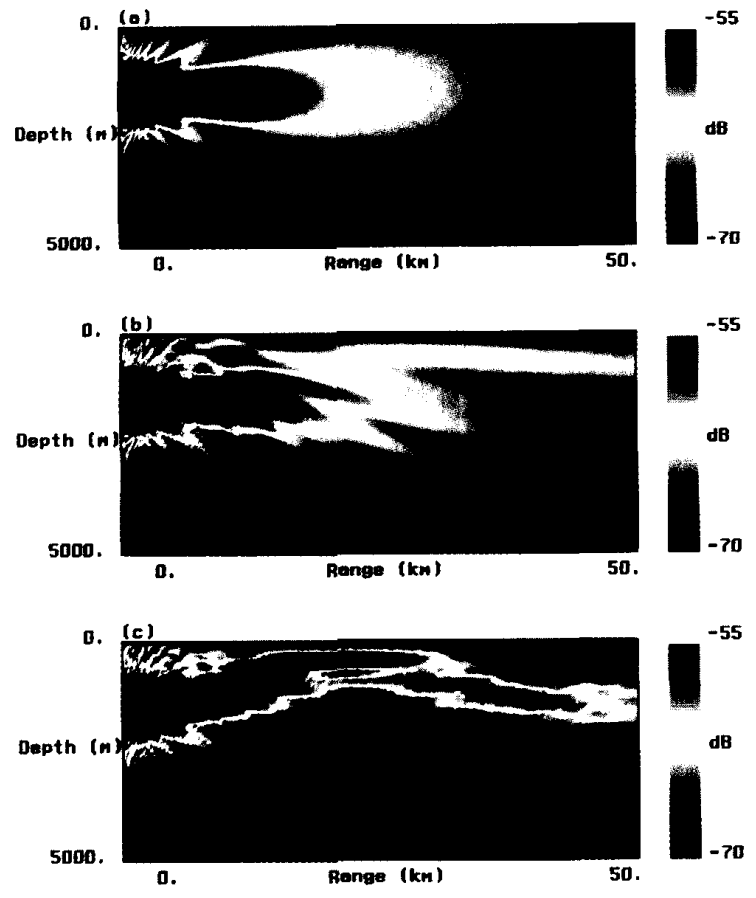

Fig. 10. Propagation of an acoustic beam using a plane-wave steering vector: (a) free space; (b) surface and bottom reflections included; (c) surface, bottom reflections and ocean refraction included.

series of sidelobes. (The sidelobes can be significantly reduced by applying an amplitude taper to the beam steering vector.) We may view this figure in two ways. Thinking of the array as a listening device, it represents the sensitivity of the array to sources at various positions.

Thinking of the array as a projector, Fig. 10 represents the field generated by an array of point sources phased to generate a horizontal beam. The term $e_{i}$ given in (21) then represents the field at $(r, z)$ due to a point source at $\left(r_{i}, z_{i}\right)$. The dot product $\boldsymbol{d}^{*} e=\sum d_{i}^{*} e_{i}$ sums the individual point sources with a phasing to generate a beam in a particular direction. In the following discussion, it is convenient to adopt this latter view of the array as a projector.

In Fig. 10(b) we include the effects of the ocean surface and ocean bottom. The sidelobes are seen to reflect off the surface and bottom yielding a clustered beam pattern. In Fig. 10 (c) we have included a sound speed profile for a particular site in the Pacific. Note how the beam lobes are refracted. It is clear from these plots that a plane-wave steering vector fails to generate a straight beam in the desired direction. If we want the beam to reach a target at a particular position then we must account for ocean refraction and purposefully steer the beam at an angle away from the source.

As mentioned earlier, acoustic models can provide a corrective "lens" for the array. For this purpose, we need waveguide steering vectors which take into account the refractive and multipath effects of the waveguide. This is easy to do. We replace the plane-wave steering vector $\boldsymbol{e}(\theta)$ by a new $\boldsymbol{e}(r, z)$ 

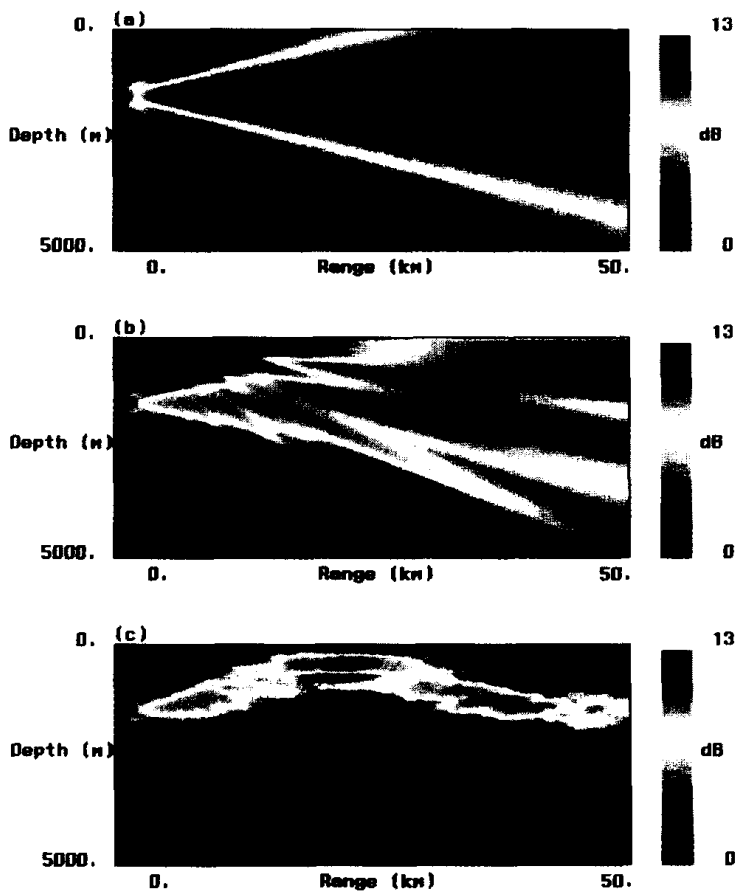

Fig. 11. Propagation of an acoustic beam using a waveguide steering vector Beam steered to range $=25 \mathrm{~km}$. depth $=$ (a) $500 \mathrm{~m}$; (b) $1500 \mathrm{~m}$; (c) $4500 \mathrm{~m}$

representing the field that would be received on the array due to a point source at $(r, z)$ and taking into account all the complex effects of the ocean channel. This is, of course, just what the acoustic models described in Section II are designed to do. For instance, we can use normal mode formula given in (15) to obtain:

$$
e_{i}(r, z) \approx \frac{i}{\sqrt{8 \pi r}} e^{-i \pi / 4} \sum_{j=1}^{\infty} Z_{j}\left(z_{i}\right) Z_{j}(z) \frac{e^{i k_{j} r}}{\sqrt{k_{j}}}
$$

This formula gives us the steering vector required to focus a beam at a particular coordinate $(r, z)$ in the waveguide. Choosing for instance, a focal range of $25 \mathrm{~km}$ range and three focal depths 500,1500 , and $4500 \mathrm{~m}$, we now obtain the three beam patterns shown in Fig. 11. Note how this waveguide steering vector directs a lobe in just the correct direction to refract to the desired focal point. It also directs sidelobes to the surface and bottom which bounce to the same focal point.

From the viewpoint of localization, there is a flaw in these beam patterns. The acoustic intensity is always strongest near the sources used to generate the beam. Or, if we think of the array as a listening device the array senses sources that are closest to it much more easily than those far way. Thus, if we identify a source by a peak in the beam response there is an intrinsic bias toward positions where the apparent source level is higher. This is easily corrected. We scale the beam response in accordance with the intensity that would be received if the source in the array were incoherent. The normalized pwoer


Fig. 12. Normalized power of an acoustic beam using a plane-wave steering vector: (a) free space; (b) surface and bottom reflections included; (c) surface, bottom reflections and ocean refraction included.

is then

$$
P(\theta)=\frac{\boldsymbol{e}^{*} R \boldsymbol{e}}{\boldsymbol{e}^{*} \boldsymbol{e} \boldsymbol{d}^{*} \boldsymbol{d}}
$$

To put this in perspective we return to the case of a plane-wave steering vector and compute the normalized power surface shown in Fig. 12. The top figure (free space) now shows a low intensity in the near field. A source in the near field generates a highly curved spherical wave front poorly matched by our plane-wave steering vector. In the far field the spherical wave is well approximated by a plane wave (with the correct angle of incidence) and the beam response is high at the correct bearing. In Figs. 12(b), (c) where the boundaries and refractive effects have been included we see similar effects.

Of greater interest is the normalized beam pattern obtained with waveguide steering vectors shown in Fig. 13. The maximum beam response now occurs precisely at the desired focal point. We see several sidelobes with high intensities however by adjusting the color scale the mainlobe at the specified focal point is easily distinguished.

We have been speaking of these plots as representing the field due an array of sources. The basic calculation is a simple dot product between two vectors $e$ and $\boldsymbol{d}$ where one of the vectors is a fixed steering vector and the other vector represents the field that would be obtained on the array due to a point source at a particular range and depth. Thus Fig. 13(a) may also be viewed as an ambiguity surface for a source located at $25 \mathrm{~km}$ range and $500 \mathrm{~m}$ depth as the array is steered to the domain of possible source positions. (This relationship 

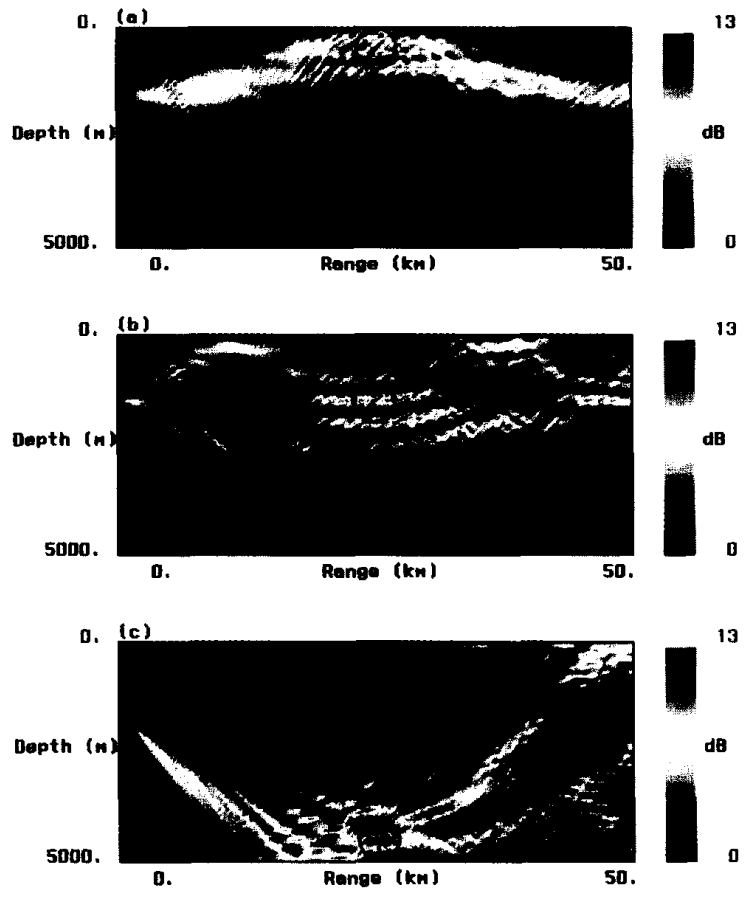

Fig. 13. Normalized power for an acoustic beam using a waveguide steering vector. Beam steered to range $=25 \mathrm{~km}$, depth $=$ (a) $500 \mathrm{~m} ;=$ (b) 1500 $\mathrm{m}$; (c) $4500 \mathrm{~m}$.

between the back-propagated field and the ambiguity surface was highlighted in [16].) The existence of a global maximum at the true source position means that the processor correctly localizes the source.

This technique of using acoustic models in the signal processing was clearly enunciated by Bucker [17] and referred to as matched-field processing. Several people proposed closely related methods, see for instance, [18] or independently arrived at the same scheme, see for instance, [19].

Matched-field processing is one of the most exciting and demanding applications of acoustic models. Both the phase and the amplitude are exploited in the signal processing. It is easy to appreciate how severe these demands are. In Fig. 13(a) we clearly see the beam focus relies on three beams. There is: 1) a direct beam that travels to the focus without striking the boundaries; 2) a bottom bounce beam; 3 ) a beam that strikes the surface, then the bottom before entering the focal point. To properly focus at the desired position the starting phase of each beam must be such that they all arrive in phase at the focal point. The source frequency is $15 \mathrm{~Hz}$ so the wavelength is approximately $100 \mathrm{~m}$. Thus, if the bottom depth is off by just $25 \mathrm{~m}$ the bottom reflected wave will arrive exactly out of phase. Small changes in the bottom properties will also cause errors in the phase of the bottom reflected wave.

The wave takes about $17 \mathrm{~s}$ to travel from the array to the focal point at $25 \mathrm{~km}$ range. A change in the sound speed of a few meters/second can also change the path length by half a

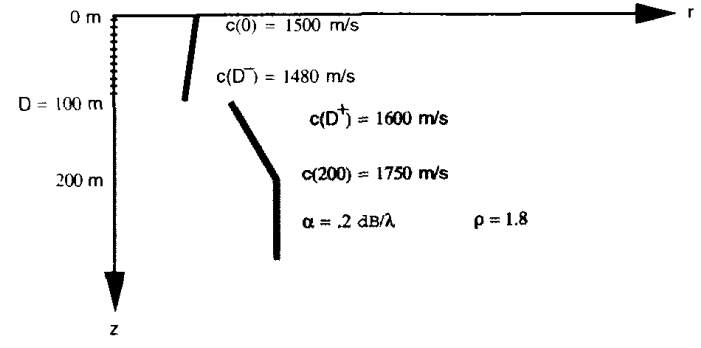

Fig. 14. Schematic of the shallow water problem.

wavelength and scramble the relative phases of different paths.

Looked at this way it would seem naive to attempt this sort of processing. On the other hand, some features will be robust. Looking at Fig. 13(a), it is clear that even with an incorrect phase, the direct, bottom and surface bounce beams will all come together somewhere near the desired position. Furthermore, even where localization fails, there may be a significant increase in detection performance.

Many authors have performed exhaustive parameter studies to quantify the importance of thse effects, see for instance [20]. A recent text by Tolstoy [21] provides a thorough review of such work. Unfortunately, useful qualitative insights are difficult to come by since the behavior of the acoustic field depends in a complicated way on so many factors such as source frequency, water depth, and source position.

In summary, conventional plane-wave beamforming uses too naive a replica of the received field in certain cases. Matched-field processing may make the opposite error of assuming too much accuracy in the acoustic model. The optimal processor must strike a compromise. This will require generating a family of replica vectors representing the spread of possible ocean SSP's, bottom types and source positions. Progress in this area is given by [22], [23].

\section{B. Broadband Localization and Back-Propagation}

While most of the matched-field work has been directed to the passive $\mathrm{CW}$ scenario it is clear that the general technique of using acoustic models to improve detection and localization is broadly applicable. We may consider passive and active $\mathrm{CW}$ processing. We may also consider passive and active broadband processing.

An interesting approach-matched signal (MESS) processing-was used by Parvulescu, Clay and coworkers at the Hudson Laboratories [24], [25]. Their approach required timedomain correlations and broadband acoustic models. At the time when the original experiments were done (1961-1963) these sorts of numerical calculations were quite difficult. Today we can study the process on a workstation quite readily. (Previous results were presented in [26].) The correlations are trivial but the broadband acoustic modeling still poses something of a burden.

The process is perhaps best introduced by example. We consider a shallow water scenario illustrated in Fig. 14. A Gaussian pulse is emanated from a source located in the middle 


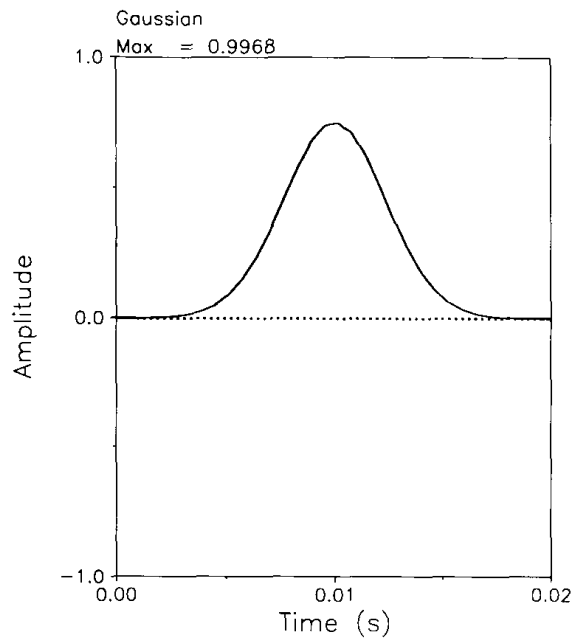

Fig. 15. Source time series for the shallow water problem.



Fig. 16. Received time series for the shallow water problem.

of the waveguide and at a range of $3 \mathrm{~km}$ from the array. The transmitted pulse is shown in Fig. 15. Note that its peak occurs at $0.01 \mathrm{~s}$. The resulting received time series is shown in Fig. 16. In an operational system this would be the received data. In our case we synthesize the data using a time domain FFP model [27].

This $3 \mathrm{~s}$ time series is then time reversed and played back through the array of hydrophones again using a broadband acoustic model. In Fig. 17 we show three snapshots of the field at times $2.5,2.99$, and $3.5 \mathrm{~s}$. We observe the back-propagate pulse coming to a focus in the second frame. The focusing occurs at a time slightly less than $3 \mathrm{~s}$. The pulse actually refocuses a hundredth of a second earlier. This corresponds to the fact that the Gaussian has its peak at one hundredth of a second.

The snapshots have all been normalized to lie between \pm 1 before plotting, however, the maximum value before
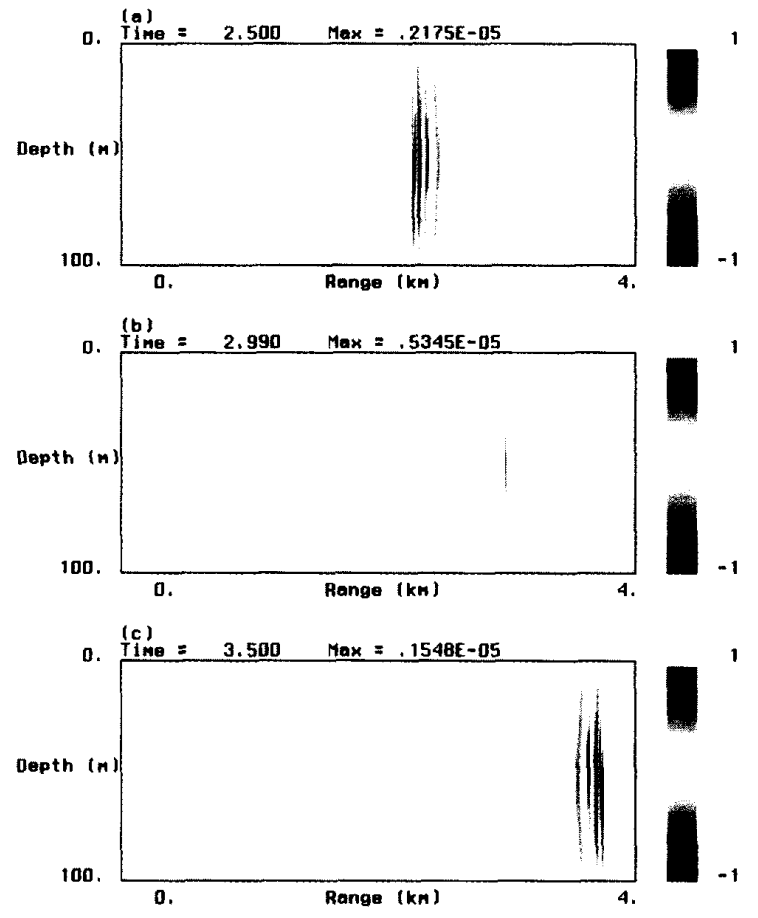

Fig. 17. Snapshots of the backpropagated field for the shallow water problem.

normalization is shown on the plot. Notice that the peak value is also highest at the refocusing time of $2.99 \mathrm{~s}$. The source is localized by searching for the position of this maximum.

An operational scenario involves measuring the receiver time series on the array and using a computer model to backpropagate the data. Just as in $\mathrm{CW}$ matched-field processing a lot is asked of the acoustic model: It must be able to duplicate the effects of the ocean waveguide. As mentioned above, when Parvulescu and Clay performed the original experiments the resources were not available to do the acoustic modeling on a computer. They therefore used a massive analog computer consisting of the ocean itself (the so-called natural lab). That is, they played the signal back through the ocean. In the vicinity of the source position they then measured the time series of the backpropagated signal and demonstrated the expected refocusing at the source position. Besides observing this in both deep and shallow water, they were also able to perform the experiment in a cluttered laboratory relying on the echoes from the boundaries of the laboratory.

In our simulation, the circumstances are reversed. The computing power is cheap and the massive analog computer is expensive to run. We therefore perform the entire experiment in silicon. A clear graphical motivation of the above process is provided in the report by Parvulescu [25]. From a mathematical point of view one is localizing the source by seeking a match between the received time series $r(t)$ and a replica of the time series. For the $\mathrm{CW}$ case we had previously used a dot product. In the time domain the obvious check for similarity 
is a correlation:

$$
P(\tau, x)=\frac{\int_{-\infty}^{\infty} r(t) e(t-\tau, x) d t}{\sqrt{\int_{-\infty}^{\infty} r^{2}(t) d t \int_{-\infty}^{\infty} e^{2}(t) d t}}
$$

Here, $e(t)$ is a replica or prediction of the received time series. We shall neglect the term in the denominator to yield an unnormalized power. This degrades the localization performance but simplifies both the numerics and our discussion.

The replica time series is given by:

$$
e(t, x)=\int_{-\infty}^{\infty} s(\tau) g(t-\tau, \boldsymbol{x}) d \tau
$$

where $s(\tau)$ is the source time series and $g(t, x)$ is the impulse response of the channel as computed by an acoustic model. Substituting this form into (27) we obtain:

$$
P(\tau, x)=\int_{-\infty}^{\infty} s(t) r^{b p}(t+\tau, x) d t
$$

where

$$
r^{b p}(t, x)=\int_{-\infty}^{\infty} r(-t) g(\tau-t, x) d \tau
$$

represents the back-propagated field, that is, the field obtained by using the reversed time series $r(-t)$ as a source. Thus, correlating the predicted and measured receiver time-series is equivalent to correlating the source time-series with the back-propagated time series.

In practice we do not know the source time-series although in principle we can construct it through deconvolution. A simple estimator is obtained by assuming the source is impulse so that we can set $s(t)=\delta\left(t-t_{0}\right)$. We then obtain,

$$
P(\tau)=r^{b p}\left(t_{0}+\tau, x\right) .
$$

This function is the back-propagated field we plotted in Fig. 17.

The source localization can be done by looking for a peak in the $P(\tau)$ or in some other measure such as the square integral of $P(\tau)$. (A variety of options are compared in [28], [29].) Note that by Parseval's theorem

$$
\int_{-\infty}^{\infty}|P(\tau)|^{2} d \tau=\left.\int_{-\infty}^{\infty} P(\omega)\right|^{2} d \omega
$$

where $P(\omega)$ is the Fourier transform of $P(\tau)$. Then, from (27) it is easy to see that

$$
P(\omega)=r(\omega)^{*} e(\omega)
$$

This is the same power function used in the previous section for a CW source. Thus, back-propagation in the time-domain is equivalent to constructing an ambiguity surface for each frequency and adding up (integrating) the individual ambiguity surfaces. Clearly, if we knew the source spectrum we would prewhiten by adding up a weighted sum of the ambiguity surfaces. In assuming a delta function for the source, we also assumed a white spectrum.

In the previous section, we discussed the scaling of the power to construct a normalized power function for $\mathrm{CW}$ matched-field processing. Similarly this back-propagation algorithm is improved by scaling $P(\tau, x)$ in accordance with the mean energy level that would be obtained by backpropagating incoherent impulses.

\section{CONCLUSIONS}

Acoustic models play an important role in the design and optimal deployment of sonar systems. In one sense, the four major model types that we described have reached a state of maturity-computations along single bearings and for single frequency sources are readily made. However, more demands are now being placed on such models. For instance, full-wave modeling of reverberant fields in a 3-D ocean environment is a challening problem. Such problems add the complexity of both broadband sources and the azimuthal variation in the environment. These factors essentially increase the dimensionality of the problem from 2-D (range-depth) to 4 D (3 space dimensions plus time). Furthermore, the reverberant or backscattered field is the component most often neglected to simplify transmission loss modeling.

Another key issue affecting acoustic models is their direct use in the signal processing algorithms. As we have illustrated, the bending of sound and the numerous bottom and surface echoes can seriously degrade standard schemes based on plane-wave beamforming. Matched-field processing problems that use acoustic models to correct for these effects are challening for many reasons. They involve repeated field calculations as a trial source is swept over the domain of possible source positions to generate waveguide replica vectors. Again, when this is done in 3-D for transient sources the computational costs are nontrivial. Lastly, optimal processors require that a deterministic ocean environment be replaced with a stochastic one recognizing the error bars on the environmental data. The acoustic models must then be run repeatedly in a Monte-Carlo fashion to construct an ensemble of acoustic fields.

\section{REFERENCES}

[1] R. J. Urick, Principles of Underwater Sound. New York: McGraw-Hill, 1983.

[2] F. B. Jensen and M. C. Ferla, "SNAP: the SACLANTCEN normal mode acoustic propagation model," SACLANTCEN SM-121, La Spezia, Italy, SACLANT Undersea Research Centre, 1979.

[3] R. Thiele, "NATO model study," SACLANT Undersea Research Rep., SR-149, 1989.

[4] F. Jensen, W. Kuperman, M. Porter, and H. Schmidt, Computational Ocean Acoustics. New York: American Institute of Physics, 1994.

[5] F. R. DiNapoli and R. L. Deavenport, "Theoretical and numerical Green's function field solution in a plane multilayered medium," $J$. Acoust. Soc. Am., vol. 67, pp. 92-105, 1980.

[6] Andreas Antoniou, Digital Filters: Analysis and Design. New York: McGraw-Hill, 1979.

[7] H. Schmidt, "SAFARI: Seismo-acoustic fast field algorithm for range independent environments. User's Guide," Rep. SR-113, SACLANT Undersea Research Centre, La Spezia, Italy, 1988.

[8] M. B. Porter, "The KRAKEN normal mode program," SACLANT Undersear Research Centre Memorandum (SM-245)/Naval Research Laboratory Mem. Rep. 6920, 1991.

[9] M. B. Porter and E. L. Reiss, "A numerical method for ocean acoustic normal modes," J. Acoust. Soc. of Am., vol. 76, no. 1, pp. 244-252, 1984.

[10] D. Lee, G. Botseas, and J. S. Papadakis, "Finite difference solutions to the parabolic equation," J. Acoust. Soc. Am., vol. 70, pp. 795-800, 1981.

[11] F. Tappert, "The parabolic equation method," in Wave Propagation and Underwater Acoustics, J. B. Keller and J. S. Papadakis, Eds. New York: Springer-Verlag, 1977 
[12] M. D. Collins, "Higher-order parabolic approximations for accurate and stable elastic parabolic equations with application to interface wave propagation," J. Acoust. Soc. Am., vol. 89, pp. 1050-1057, 1991.

[13] W. A. Kuperman, M. B. Porter, J. S. Perkins, and R. B. Evans, "Rapid computation of acoustic fields in three-dimensional ocean environments," J. Acoust. Soc. Am., vol. 89, no. 1, pp. 125-133, 1991.

[14] D. A. Gershfeld and F. Ingenito, "Optimum depth of propagation in shallow water," Naval Research Laboratory Rep. 8741, 1983.

[15] C. M. Ferla and M. B. Porter, "Receiver-depth selection for passive sonar systems," IEEE J. of Oceanic Engineering, vol. 16, pp. 267-278, 1991.

[16] L. Nghiem-Phu, F. D. Tappert, and S. C. Daubin, "Source localization by CW acoustic retrogration," Proceedings of a Workshop on Acoustic Source Localization, internal NRL rep. R. Fizell, Ed., 1985

[17] H. P. Bucker, "Use of calculated sound fields and matched-field detection to locate sound sources in shallow water," J. Acoust. Soc. Am., vol. 59 , pp. 368-373, 1976.

[18] C. S. Clay, "Waveguides, arrays and filters," Geophysics, vol. 31, pp. 501-506, 1966.

[19] R. M. Heitmeyer, W. B. Moseley, and R. G. Fizell, "Full field ambiguity function processing in a complex shallow-water environment," in High Resolution Spatial Processing in Underwater Acoustics, by R. A Wagstaff and A. B. Baggeroer, Eds., NORDA proceeding, (1983).

[20] M. B. Porter, R. L. Dicus, and R. Fizell, "Simulations of matched field processing in a deep-water pacific environment," IEEE $J$. of Oceanic Engineering, vol. OE-12, pp. 173-181, 1987.

[21] A. Tolstoy, Matched Field Processing for Underwater Acoustics, World Scientific, 1993.

[22] H. Schmidt, A. B. Baggeroer, W. A. Kuperman, and E. K. Sheer "Environmentally tolerant beamforming for high-resolution matched field processing: Deterministic mismatch," J. Acoust. Soc. Am., vol. 88 pp. 1802-1810, 1990

[23] A. M. Richardson and L. W. Nolte, "A posteriori probability source localization in an uncertain sound speed, deep ocean environment," $J$. Acoust. Soc. Am., vol. 89, pp. 2280-2284, 1991.
[24] A. Parvulescu, "Signal detection in a multipath medium by M.E.S.S. processing," J. Acoust. Soc. Am., vol. 33, p. 1674, 1961.

[25] A. Parvulescu, "Acoustical signal processing by the deep ocean," Hawail Institute of Geophysics Rep. 407, 1971.

[26] M. B. Porter and F. Jensen, "Acoustic pulse propagation in ocean waveguides," Proceedings of the Transients Workshop. Naval Research Laboratory pub. 152-5100, D. Bradley, Ed., 1989.

[27] M. B. Porter, "The time-marched FFP for modeling acoustic pulse propagation," J. Acoust. Soc. Am., vol. 87, no. 5, pp. 2013-2023, 1990

[28] L. N. Frazer and P. I. Pecholcs, "Single-hydrophone localization," $J$. Acoust. Soc. Am., vol. 88, pp. 995-1002, 1990.

[29] E. K. Westwood, "Broadband matched-field source localization," $J$. Acoust. Soc. Am., vol. 91, pp. 2777-2789, 1992

Michael B. Porter was born in Quebec, Canada, on September 19, 1958. He received the B.S. degree in applied mathematics from the California Institute of Technology, Pasadena, CA in 1979. In 1984 he received the Ph.D. degree in engineering science and applied mathematics from Northwestern University, Evanston, IL.

From 1983 to 1985 he was a scientist at the Naval Ocean Systems Center, San Diego, CA, where he performed research in numerical modeling of antennas, optical fibers, sonar transducers, and ocean acoustics problems. From 1985 to 1987 he was employed as a research physicist at the Naval Research Laboratory in Washington, D.C. At NRL his interest included matched-field processing and computational acoustics. From 1987-1991 he continued research in these areas a sa senior scientist at the SACLANT Undersear Research Centre (NATO) in La Spezia, Italy. He is presently a Professor of Mathematics at the New Jersey Institute of Technology.

Dr. Porter is a member of the Acoustical Society of America and the Society for Industrial and Applied Mathematics. He is the recipient of the A. B. Wood Medal (1991) from the Institute of Acoustics and an Associate Editor for the Journal of the Acoustical Society of America (Underwater Sound). 